\title{
CONTROLLED HETEROGENEOUS COLLECTION: THE ROLE OF OCCUPATION NUMBERS
}

\author{
A. GERARDI *** AND \\ P. TARDELLI, ${ }^{* * * *}$ Universitá dell'Aquila
}

\begin{abstract}
A controlled heterogeneous collection of identical items is presented. According to their level of wear and tear, they are divided into a finite number of classes and the partition of the collection is allowed to change over time. A suitable exchangeability assumption is made to preserve the property that the items be identical. The role of the occupation numbers is investigated and a filtering problem is set up, where the observation is the cardinality of a particular class. A control on the dynamics of the items is introduced, and the existence of an optimal control is proved. A discrete-time approximation for the separated problem, which is a finite-dimensional one, is performed. As a consequence, an approximation for the value function is given.
\end{abstract}

Keywords: Heterogeneous population; exchangeability; stochastic filtering; optimal stochastic control

2000 Mathematics Subject Classification: Primary 93E11; 93E20

Secondary $60 \mathrm{H} 30 ; 60 \mathrm{~J} 75$

\section{Introduction}

We construct a continuous-time model to describe the dynamics of a collection of identical items, $\left\{U_{1}, \ldots, U_{N}\right\}$. At any time $t \in \mathbb{R}^{+}$, these items are divided into different classes, $\left\{C_{1}(t), \ldots, C_{d}(t)\right\}$, according to their level of wear. As time goes on, the partition of the collection can change, since the level of wear of any single item can increase or decrease, under some kind of maintenance. In every class each item can definitively break down. The partition of the collection cannot be observed and the observation is the number of broken items up to time $t$. Next, we introduce the class $C_{0}(t)$ of the items failed up to time $t$ so that the observation coincides with the cardinality of this class.

The model just described is a generalization of that presented in [14] and [15]. There, the unobservable partition of the population was not supposed to change with time, the particles could only die, and the law of the lifetimes, given the partition of the population, was given as data.

In place of this, in this paper, as in [13] and [27], in order to allow the partition to change over time, at every time $t$, the class of each item is defined by introducing a stochastic process $Z_{t}=\left\{Z_{t}^{i}\right\}_{1 \leq i \leq N}$ such that $Z_{t}^{i}=k$ if and only if $U_{i} \in C_{k}(t)$ for $k=0,1, \ldots, d$. The dynamics of this process have to obey some constraints. First, as supposed in [27], in order to take into account the dynamics of the model and the fact that the particles are identical, an exchangeability assumption on the finite-dimensional distribution of $Z_{t}$ is made. Moreover, we

Received 4 October 2006.

* Postal address: Dipartimento di Ingegneria Elettrica e dell'Informazione, Universitá dell' Aquila, 67040 Poggio di Roio, Italy.

** Email address: gerardi@ing.univaq.it

*** Email address: tardelli@ing.univaq.it 
define $X_{t}$, the vector of the occupation numbers, whose components are the cardinality of each class, and $Y_{t}$, the number of failures up to time $t$. Under a second suitable assumption, the pairstate observation, $\left(X_{t}, Y_{t}\right)$, is a pure-jump Markov process. Roughly speaking, this assumption reduces to supposing that the dynamics of the process $Z_{t}$ depend only on the crowding of each class. These topics are discussed in Section 2.

In order to complete the discussion on the model introduced in [13], in this paper we introduce a control on the dynamics of the process $\left(X_{t}, Y_{t}\right)$, assuming that $u_{t}$, the control process, is adapted to the observed history $\mathcal{F}_{t}^{Y}=\sigma\left\{Y_{s} ; s \leq t\right\}$. In Section 3 we define a cost function in terms of the running cost, which represents the maintenance cost, and the terminal cost, which represents the knowledge gained during the observation time interval $[0, T]$. Hence, we are faced with a partially observed control problem. There is a wide body of literature about partially observable optimal control problems, mainly in the diffusive case, and relaxed controls are introduced both in deterministic and stochastic control problems (see [8], [11], [22], [25], and the references therein). Control problems involving Markov chains with counting observations have also been largely investigated. For instance, in [9], [16], [19], and the references therein, a separation result is reached by using the reference probability method and by establishing the dynamic programming equation for the control problem.

In this paper we follow a different approach, inspired by [8] and [25]. Let us quote [4], [5], and [7] also. We observe that in [25] common jump times between the state and observations are not allowed. In [4] and [5], the state and observations have the same jump times, since the observations are just the process counting all the jumps of the state. In [4], only the equivalence between the partially observed problem and the separated problem is proved. Essentially, the main line of this paper is in some sense driven by [7], in which a quite general model was considered. The main difference is that the model investigated in this paper allows us to obtain better results in an easier and direct way.

As in [7], we introduce the associated separated control problem, where the state is the pair filter-observations: namely, the first component is a probability-measure-valued process which has to obey the controlled filtering equation. A first result, presented in Section 4, is the proof of the equivalence between the partially observable control problem and the separated control problem. This means that the infimum expected cost of the separated problem is equal to the infimum expected cost of the original problem. Equivalence is an essential tool to prove the existence of an optimal (relaxed) strategy and it is based on the property of uniqueness for the solution of the filtering equation. The existence of an optimal strategy is obtained in Section 5, by a compactification method. Such a method is not a constructive one. Since in our model the filter turns out to be finite-dimensional, we construct, in Section 6, a sequence of discrete-time processes such that their continuous-time interpolation [23], [24, Chapter 10] converges to the original process. This construction also provides an approximation of the value function.

\section{Occupation numbers}

We study a finite or countable collection $\mathcal{P}=\left\{U_{j}\right\}_{j \geq 1}$, where $U_{j}$ are given items. Choosing a positive integer $N$, let $\mathcal{P}_{N}=\left\{U_{j}\right\}_{j=1, \ldots, N}$ be a finite subpopulation of $\mathcal{P}$. We consider $\mathcal{P}_{N}$ to be heterogeneous in the sense that its elements are of $d$ different types, labeled by the natural numbers $1, \ldots, d$. At any time, every item can change its type, or definitively break down. Given $t \in \mathbb{R}^{+}$, let $C_{k}(t)$ be the subset of all items of type $k, k=1, \ldots, d$, at time $t$, and let $C_{0}(t)$ be the class of items broken down up to time $t$. Thus, $\mathcal{P}_{N}=\bigcup_{k=0,1, \ldots, d} C_{k}(t)$. We suppose that the dynamics of the items are random. Then, on a probability model $(\Omega, \mathcal{F}, \mathrm{P})$, we assume that events of the form $\left\{U_{i} \in C_{k}(t)\right\}$ for $i=1, \ldots, N$ are measurable. 
The situation we are faced with is, for instance, a collection of industrial items under stress. As time goes on, the items are subjected to some kind of maintenance and this can change their condition. Alternatively, we could also consider a population of individuals affected by the same kind of disease, who are receiving medical treatment.

In order to define each item's type, let us introduce the random variable $Z_{t}=\left\{Z_{t}^{i}\right\}_{i=1, \ldots, N}$ such that, for all $t \in \mathbb{R}^{+}$and $k=0,1, \ldots, d$,

$$
Z_{t}^{i}=k \quad \Longleftrightarrow \quad U_{i} \in C_{k}(t), \quad i=1, \ldots, N
$$

Therefore, for all $t \in \mathbb{R}^{+}, Z_{t}^{i}$ is a random variable taking values in $\{0,1, \ldots, d\}$ and $Z_{t}=$ $\left\{Z_{t}^{i}\right\}_{i=1, \ldots, N}$ is a random vector taking values in $\{0,1, \ldots, d\}^{N}$.

We note that $C_{0}(t)$ is in some sense an 'absorbing' class, because if an item $U_{i}$ goes into $C_{0}(t)$ then it stays there definitively. Thus, we have the following assumption.

Assumption 2.1. For all $i=1, \ldots, N$, we assume that

$$
Z_{s}^{i}=0 \quad \Longrightarrow \quad Z_{t}^{i}=0 \text { for all } t \geq s \text { almost surely (a.s.). }
$$

In the construction of the model, as in [13] and [27], we have to take into account the dynamics of the model and the fact that different items can have different labels even if they are considered undistinguishable. Therefore, we introduce an exchangeability property, which involves the finite-dimensional distribution of $Z_{t}=\left(Z_{t}^{1}, \ldots, Z_{t}^{N}\right)$.

Assumption 2.2. For all $n \geq 1$, all $t_{1}, \ldots, t_{n} \in \mathbb{R}^{+}$with $t_{1} \leq \cdots \leq t_{n}$, all permutations $\beta$ of the set $\{1, \ldots, N\}$, and all $k^{(1)}, \ldots, k^{(n)} \in\{0,1, \ldots, d\}^{N}$,

$$
\mathrm{P}\left(Z_{t_{1}}=k^{(1)}, \ldots, Z_{t_{n}}=k^{(n)}\right)=\mathrm{P}\left(Z_{t_{1}}=\beta k^{(1)}, \ldots, Z_{t_{n}}=\beta k^{(n)}\right),
$$

where, for $k^{(i)}=\left\{k_{1}^{(i)}, \ldots, k_{N}^{(i)}\right\} \in\{0,1, \ldots, d\}^{N}, \beta k^{(i)}=\left\{k_{\beta_{1}}^{(i)}, \ldots, k_{\beta_{N}}^{(i)}\right\}, i=1, \ldots, n$.

Roughly speaking, this last assumption is, in some sense, an exchangeability property of the trajectories of the process $Z$. Furthermore, we note that, in particular, Assumption 2.2 implies that the sequence $\left(Z_{t}^{1}, \ldots Z_{t}^{N}\right)$ is exchangeable for any $t$, that is, for all $a_{1}, \ldots, a_{N} \in$ $\{0,1, \ldots, d\}$ and all finite permutations $\beta$ of the indices,

$$
\mathrm{P}\left(Z_{t}^{1}=a_{1}, \ldots, Z_{t}^{N}=a_{N}\right)=\mathrm{P}\left(Z_{t}^{1}=a_{\beta(1)}, \ldots, Z_{t}^{N}=a_{\beta(N)}\right),
$$

and this implies that (2.2) is a generalization of the exchangeability property introduced in [14] and [15]. There, condition (2.3) was sufficient to obtain the exchangeability property for the joint distribution of the lifetimes. In our context, this is not the case and the most important consequence of condition (2.2) is Proposition 2.1, below, which was proved in [13].

Proposition 2.1. Under conditions (2.1) and (2.2), the sequence $\left\{T_{i}\right\}_{i \geq 1}$, where $T_{i}$ is the lifetime of $U_{i}$, defined as

$$
T_{i}=\inf \left\{t \in \mathbb{R}^{+}: Z_{t}^{i}=0\right\}, \quad i=1, \ldots, N,
$$

is a family of exchangeable random variables.

We note that, in many models, the dynamics of the process $Z_{t}$ depends only on the crowding of each class. For instance, we can consider a multidimensional queue, where the dynamics of the arrival and departure processes can depend only on the number of customers waiting. Thus, we will consider this kind of situation, which brings us to the following setup. Let us note that this is the main difference between the model presented in this paper and that discussed in [13]. 
First, as in [27], we define the occupation numbers representing the cardinality of each class.

Definition 2.1. For $i=1, \ldots, d$ and $z=z^{1}, \ldots, z^{N}$, let $\Phi_{i}(z)=\sum_{j=1}^{N} \mathbf{1}_{\left\{z^{j}=i\right\}}$ and, for $t \in \mathbb{R}^{+}$, we define $X_{t}^{i}:=\# C_{i}(t)=\Phi_{i}\left(Z_{t}\right)$ to be the family of occupation numbers and $Y_{t}:=\# C_{0}(t)=\Phi_{0}\left(Z_{t}\right)$ to be the number of items already dead up to time $t$. Setting $\Phi=$ $\left(\Phi_{1}, \ldots, \Phi_{d}, \Phi_{0}\right)$ and $X_{t}=\left(X_{t}^{1}, \ldots, X_{t}^{d}\right)$, we obtain $\left(X_{t}, Y_{t}\right)=\Phi\left(Z_{t}\right)$.

Thus, the process $\left(X_{t}, Y_{t}\right)$ takes values in

$$
\mathcal{K}:=\left\{\left(x_{1}, \ldots, x_{d}, y\right): x_{i}, y \in \mathbb{N} \cup\{0\} \text { for all } i ; x_{1}+\cdots+x_{d}+y=N\right\},
$$

while the process $X_{t}$ takes values in $\mathcal{X}:=\left\{\left(x_{1}, \ldots, x_{d}\right): x_{i} \in \mathbb{N} \cup\{0\}\right.$ for all $i, x_{1}+\cdots+x_{d} \leq$ $N$ \}. Furthermore, condition (2.1) implies that $Y_{t}$ is, a.s., a nondecreasing function with respect to $t$.

In order to investigate the relation between $\mathcal{L}(Z)$, the law of $Z$, and $\mathcal{L}(X, Y)$, the joint law of $X$ and $Y$, let us observe that $\Phi$ is a deterministic function which is not necessarily one-to-one. Thus, for all $n$ and all $s^{(1)}, \ldots, s^{(n)} \in \mathcal{K}$,

$$
\begin{aligned}
\mathrm{P}\left(\left(X_{t_{1}}, Y_{t_{1}}\right)=s^{(1)}, \ldots,\left(X_{t_{n}}, Y_{t_{n}}\right)=s^{(n)}\right) & =\mathrm{P}\left(\Phi\left(Z_{t_{1}}\right)=s^{(1)}, \ldots, \Phi\left(Z_{t_{n}}\right)=s^{(n)}\right) \\
& =\mathrm{P}\left(Z_{t_{1}} \in \Phi^{-1}\left(s^{(1)}\right), \ldots, Z_{t_{n}} \in \Phi^{-1}\left(s^{(n)}\right)\right) .
\end{aligned}
$$

Therefore, given $\mathcal{L}(Z), \mathcal{L}(X, Y)$ is known, but, instead, in general, given $\mathcal{L}(X, Y), \mathcal{L}(Z)$ is known just for a fixed $t$. The proof of the following proposition is given in [14].

Proposition 2.2. For $k \in\{0,1, \ldots, d\}^{N}$ and all $t \in \mathbb{R}^{+}$, if (2.3) holds then

$$
\mathrm{P}\left(Z_{t}=k\right)=\mathrm{P}\left(Z_{t}^{1}=k_{1}, \ldots, Z_{t}^{N}=k_{N}\right)=\frac{\Phi_{0}(k) ! \Phi_{1}(k) ! \cdots \Phi_{d}(k) !}{N !} \mathrm{P}\left(\left(X_{t}, Y_{t}\right)=\Phi(k)\right) .
$$

We generalize Proposition 2.2 under the following further assumption, which we introduce in order to ensure that the dynamics of the process $Z_{t}$ depend only on the crowding of each class. More precisely, (2.5), below, guarantees equal probability for events having the same occupation numbers and preserving the absorbing property of the class $C_{0}(t)$. Consequently, $\mathcal{L}(Z)$ is known, given $\mathcal{L}(X, Y)$.

Assumption 2.3. For $h, \tilde{h} \in\{0,1, \ldots, d\}^{N}$, let

$$
\gamma(h, \widetilde{h}):=\prod_{i=1}^{H}\left(\mathbf{1}_{\left\{h_{i} \neq 0\right\}}+\mathbf{1}_{\left\{h_{i}=0, \widetilde{h}_{i}=0\right\}}\right) .
$$

If, for all $n>0$, all $t_{1} \leq \cdots \leq t_{n}$, all $\left(h^{(1)}, \ldots, h^{(n)}\right)$, and all $\left(h^{\prime(1)}, \ldots, h^{\prime(n)}\right), h^{(i)}, h^{\prime(i)} \in$ $\{0,1, \ldots, d\}^{N}$ such that

(i) $\Phi\left(h^{(i)}\right)=\Phi\left(h^{\prime(i)}\right), i=1, \ldots, n$,

(ii) $\gamma\left(h^{(i)}, h^{(i+1)}\right)=\gamma\left(h^{\prime(i)}, h^{\prime(i+1)}\right), i=1, \ldots, n-1$,

then let us assume that

$$
\mathrm{P}\left(Z_{t_{1}}=h^{(1)}, \ldots, Z_{t_{n}}=h^{(n)}\right)=\mathrm{P}\left(Z_{t_{1}}=h^{\prime(1)}, \ldots, Z_{t_{n}}=h^{(n)}\right) .
$$


Moreover, choosing $h^{\prime(i)}=\beta h^{(i)}$ for $i=1, \ldots, n$ and all permutations $\beta$ of the set $\{1, \ldots, N\}$, Assumption 2.3 implies (2.2), while the contrary implication does not hold. The following proposition is readily obtained by combinatorial techniques.

Proposition 2.3. Under Assumption 2.3, for $n \geq 1$, all $t_{1} \leq \cdots \leq t_{n}$, and all $h^{(1)}, \ldots$, $h^{(n)} \in\{0,1, \ldots, d\}^{N}$,

$$
\begin{aligned}
& \mathrm{P}\left(Z_{t_{1}}=h^{(1)}, \ldots, Z_{t_{n}}=h^{(n)}\right) \\
& \quad=A\left(h^{(1)}, \ldots, h^{(n)}\right) \mathrm{P}\left(\left(X_{t_{1}}, Y_{t_{1}}\right)=\Phi\left(h^{(1)}\right), \ldots,\left(X_{t_{n}}, Y_{t_{n}}\right)=\Phi\left(h^{(n)}\right)\right),
\end{aligned}
$$

where

$$
\begin{aligned}
A\left(h^{(1)}, \ldots, h^{(n)}\right) & \\
= & \frac{\Phi_{0}\left(h^{(1)}\right) ! \Phi_{1}\left(h^{(1)}\right) ! \cdots \Phi_{d}\left(h^{(1)}\right) !}{H !} \\
& \times \prod_{j=1}^{n-1} \frac{\left(\Phi_{0}\left(h^{(j+1)}\right)-\Phi_{0}\left(h^{(j)}\right)\right) ! \Phi_{1}\left(h^{(j+1)}\right) ! \cdots \Phi_{d}\left(h^{(j+1)}\right) !}{\left(H-\Phi_{0}\left(h^{(j)}\right)\right) !} \gamma\left(h^{(j)}, h^{(j+1)}\right) .
\end{aligned}
$$

Next, we investigate the Markov property for the processes $Z_{t}$ and $\left(X_{t}, Y_{t}\right)$.

If we assume that $Z_{t}$ is a Markov process, this, in general, does not imply that $\left(X_{t}, Y_{t}\right)$ is a Markov process. On the other hand, under Assumption 2.3, we have the following quite intuitive property proved in Proposition 3.5 of [27].

Proposition 2.4. Under Assumption 2.3, $\left(X_{t}, Y_{t}\right)$ is a Markov process if and only if $Z_{t}$ is a Markov process.

The Markov property is the main tool used in establishing the existence of the processes involved in this model and, in particular, allows us to construct $X_{t}$ as the solution of a suitable martingale problem, as we will see in the next section.

\section{Setting of the control problem}

From this point on, we focus attention on the situation in which Assumption 2.3 holds true and the pair $\left(X_{t}, Y_{t}\right)$ is a partially observed, controlled Markov process on a finite horizon $[0, T]$. We are looking for $\mathcal{F}_{t}^{Y}$-predictable controls taking values in $U$, a finite subset of $\mathbb{R}^{n}$, where $\mathcal{F}_{t}^{Y}=\sigma\left\{Y_{s}, s \leq t\right\}$.

In the model we are looking for, the state process, $X_{t}$, jumps and we can observe just some of its jumps. For $X_{t}=x$ and under a constant control $u \in U$ with a rate $\lambda_{0}^{u}(x), X_{t}$ jumps alone following a transition function $\mu_{0}^{u}\left(x, x^{\prime}\right)$. Alternatively, with rate $\lambda_{1}^{u}(x), X_{t}$ jumps following a transition function $\mu_{1}^{u}\left(x, x^{\prime}\right)$ and in this case, setting $|x|=x_{1}+\cdots+x_{d}, Y_{t}:=N-\left|X_{t}\right|$ increases. Finally, this is our choice: only the second kind of jumps are registered by the observations process $Y_{t}$. This reduces to supposing that the state $X_{t}$ is a partially observable, pure-jump process, controlled using only the information given by the process $Y_{t}$.

In the sequel we will assume that $Y_{t}$ is a counting process. If this is not the case, we could introduce, as in the uncontrolled model discussed in [13] and [27], the multivariate point process $W(t)=\left(W^{1}(t), \ldots, W^{N}(t)\right)$ defined as

$$
W^{j}(t):=\sum_{i \geq 1} \mathbf{1}_{\left\{\tau_{i} \leq t\right\}} \mathbf{1}_{\left\{Y\left(\tau_{i}\right)=j\right\}}, \quad j=1, \ldots, N,
$$


where $\left\{\tau_{i}\right\}_{i \geq 1}$, the order statistics of the lifetimes, gives us the jump times of $Y$. The process $W^{j}(t)$ counts the number of jumps bringing $Y(t)$ on $j$. This gives us some technical complication in writing the filtering equation. Therefore, for the sake of simplicity, we assume that $Y_{t}$ is a counting process and that, under the action of a constant control $u \in U$, the process $X_{t}$ is a Markov process with generator $L^{u} f(x)=L_{0}^{u} f(x)+L_{1}^{u} f(x)$, where

$$
\begin{aligned}
& L_{0}^{u} f(x)=\lambda_{0}^{u}(x) \sum_{x^{\prime} \in \mathcal{X}}\left(f\left(x^{\prime}\right)-f(x)\right) \mu_{0}^{u}\left(x, x^{\prime}\right), \\
& L_{1}^{u} f(x)=\lambda_{1}^{u}(x) \sum_{x^{\prime} \in \mathcal{X}}\left(f\left(x^{\prime}\right)-f(x)\right) \mu_{1}^{u}\left(x, x^{\prime}\right) .
\end{aligned}
$$

The functions $\lambda_{0}^{u}(x)$ and $\lambda_{1}^{u}(x)$ are necessarily bounded and measurable on $U \times \mathcal{X}$, as any other real-valued function defined on a finite space. Moreover,

$$
\lambda_{i}^{u}(x)=0 \quad \text { if } \quad|x|=0 \quad \text { for } i=0,1 .
$$

We suppose that $\lambda_{1}^{u}\left(X_{t}\right)>0$ until $\tau_{N}$, the first time such that $Y_{t}=N$. Obviously, $\tau_{N}$ is an $\widetilde{F}_{t}^{Y}$-stopping time, i.e. $\tau_{N}$ is an observable random variable and, for $t \geq \tau_{N},\left|X_{t}\right|=0$ and $Y_{t}=N$.

Furthermore, we assume that, for any $u \in U, \mu_{0}^{u}\left(x, x^{\prime}\right)$ and $\mu_{1}^{u}\left(x, x^{\prime}\right)$ verify the following properties:

$$
\mu_{0}^{u}\left(x, x^{\prime}\right)=\mu_{0}^{u}\left(x, y, x^{\prime}\right) \mathbf{1}_{\left\{\left|x^{\prime}\right|=|x|\right\}}=\mu_{0}^{u}\left(x, N-|x|, x^{\prime}\right) \mathbf{1}_{\left\{\left|x^{\prime}\right|=|x|\right\}}
$$

and

$$
\mu_{1}^{u}\left(x, x^{\prime}\right)=\mu_{1}^{u}\left(x, x^{\prime}\right) \mathbf{1}_{\left\{\left|x^{\prime}\right|+1=|x|\right\}} \mathbf{1}_{\{|x|>0\}},
$$

which guarantee that $\left(X_{t}, Y_{t}\right) \in \mathcal{K}$ (see (2.4)) whenever the initial conditions do. Let the set of admissible controls, $U$, consist of all $U$-valued $\mathcal{F}_{t}^{Y}$-predictable processes. Therefore, $X_{t}$ is a controlled jump process taking values in $\mathcal{X}$ and the observations process, $Y_{t}$, counts all the jumps of the process $\left|X_{t}\right|$.

For any admissible control, $u_{t}$, we will construct the process $X_{t}$ as a solution of the controlled martingale problem for $L^{u_{t}}$, with initial condition $v_{0}$, for each $v_{0} \in \Pi(\mathcal{X})$, the space of the probability measures on $\mathcal{X}$ (henceforth abbreviated as $\operatorname{MgP}\left(L^{u_{t}}, v_{0}\right)$ ). This reduces to finding, given $v_{0} \in \Pi(\mathcal{X})$, a filtered space, $\left(\Omega,\left\{\mathcal{F}_{t}\right\}\right)$, a process $X_{t}$ on it, and, for any admissible control $u_{t} \in \mathcal{U}$, a probability measure $\mathrm{P}$ such that $\mathcal{L}\left(X_{0}\right)=v_{0} \mathrm{P}$-a.s. and, for any real-valued function $f$ defined on $\mathcal{X}$, such that the process

$$
f\left(X_{t}\right)-f\left(X_{S}\right)-\int_{s}^{t} L^{u_{r}} f\left(X_{r}\right) \mathrm{d} r \quad \text { for all } s \leq t
$$

is a $\left(\mathrm{P}, \mathscr{F}_{t}\right)$-martingale. We note that in our frame, on the space $\left(\Omega,\left\{\mathcal{F}_{t}\right\}, \mathrm{P}\right)$, for any $X_{t}$ solution to the $\operatorname{MgP}\left(L^{u_{t}}, v_{0}\right), Y_{t}=N-\left|X_{t}\right|$ is a point process with $\left(P, \mathcal{F}_{t}\right)$-intensity $\lambda_{1}^{u_{t}}\left(X_{t}\right)$, i.e. $Y_{t}-\int_{0}^{t} \lambda_{1}^{u_{s}}\left(X_{s}\right) \mathrm{d} s$ is a $\left(\mathrm{P}, \mathcal{F}_{t}\right)$-martingale. This implies that a $U$-valued $\mathcal{F}_{t}^{Y}$-predictable process [1, p. 309] has the structure

$$
u_{t}=\sum_{n \geq 0} u_{n}\left(\tau_{1}, \ldots, \tau_{n}, t\right) \mathbf{1}_{\left(\tau_{n}, \tau_{n+1}\right]}(t),
$$

where $\left\{u_{n}\right\}_{n \geq 0}$ are $U$-valued measurable functions and $\left\{\tau_{k}\right\}_{k=1, \ldots, N}$ denotes the jumps times of $Y_{t}$ (we assume that $\tau_{0}=0$ and that $u_{0}$ depends only on $t$ ).

This means that an admissible control $u_{t}$ is completely characterized by the sequence $\left\{u_{n}\right\}_{n \geq 0}$. 
Proposition 3.1. For any initial measure $v_{0} \in \Pi(\mathcal{X})$, let the sequence $\left\{u_{n}\right\}_{n \geq 0}$ be a family of measurable functions $u_{n}:[0, \infty)^{n+1} \rightarrow U, n \geq 0$. Under our setting, there exists a solution of the controlled $\operatorname{MgP}\left(L^{u_{t}}, v_{0}\right)$, where $u_{t}$ is given by (3.1).

Proof. The proof is a slight modification of that given in [4]. It is based on a result given in [17] related to the representation of the predictable projection of the random measures associated to jump processes. We confine ourselves to recalling the construction of the solution.

Let $(\Omega, \mathcal{F})$ be the canonical space of the marked point processes and, on this space, let $\left(\widetilde{T}_{n}, \widetilde{X}_{n}\right)_{n \geq 0}$ be the canonical process. We define $X_{t}$ as

$$
X_{t}= \begin{cases}\widetilde{X}_{n} & \text { if } \widetilde{T}_{n} \leq t<\widetilde{T}_{n+1} \\ \eta & \text { if } t \geq \widetilde{T}_{\infty}\end{cases}
$$

where $\widetilde{T}_{\infty}=\lim _{n \rightarrow \infty} \widetilde{T}_{n}, \eta$ is an extra point outside $\mathcal{X}$, and $\mathcal{F}_{t}=\sigma\left\{X_{s}, s \leq t\right\}$. Let $\mathrm{P}=$ $\mathrm{P}\left(\left\{u_{n}\right\}, v_{0}\right)$ be the unique probability measure [17] such that

$$
\begin{aligned}
v(\mathrm{~d} t, \mathrm{~d} x)=\sum_{n \geq 0} \sum_{x^{\prime} \in \mathcal{X}} & \left(\lambda_{0}^{u_{n}\left(\widetilde{T}_{1}, \ldots, \widetilde{T}_{n}, t\right)}\left(\widetilde{X}_{n}\right) \mu_{0}^{u_{n}\left(\widetilde{T}_{1}, \ldots, \widetilde{T}_{n}, t\right)}\left(\widetilde{X}_{n}, x^{\prime}\right) \delta_{\left\{\left|x^{\prime}\right|\right\}}(\mathrm{d}|x|)\right. \\
& \left.+\lambda_{1}^{u_{n}\left(\widetilde{T}_{1}, \ldots, \widetilde{T}_{n}, t\right)}\left(\widetilde{X}_{n}\right) \mu_{1}^{u_{n}\left(\widetilde{T}_{1}, \ldots, \widetilde{T}_{n}, t\right)}\left(\widetilde{X}_{n}, x^{\prime}\right) \delta_{\left\{\left|x^{\prime}\right|-1\right\}}(\mathrm{d}|x|)\right) \\
& \times \delta_{\left\{x^{\prime}\right\}}(\mathrm{d} x) \mathbf{1}_{\left(\widetilde{T}_{n}, \widetilde{T}_{n+1}\right]}(t) \mathrm{d} t
\end{aligned}
$$

is the predictable projection of

$$
m(\mathrm{~d} t, \mathrm{~d} x)=\sum_{n \geq 1} \delta_{\left\{\widetilde{T}_{n}, \widetilde{X}_{n}\right\}}(\mathrm{d} t, \mathrm{~d} x) \mathbf{1}_{\left\{\widetilde{T}_{n}<\infty\right\}} .
$$

Then, the process counting all the jumps of $X_{t}, \sum_{n \geq 1} \mathbf{1}_{\left[\widetilde{T}_{n},+\infty\right)}(t)$, is a point process with finite first moment and this implies that $X_{t}$ is a nonexplosive process (i.e. $\widetilde{T}_{\infty}=\infty$ ). This completes the proof.

Proposition 3.1 allows us to construct the partially observable (p.o.) control, $\left(\Omega, \mathcal{F}_{t}, \mathrm{P}, X_{t}\right.$, $\left.Y_{t}, u_{t}, v_{0}, 0\right)$, where the control $u_{t}$ is defined by the sequence $\left\{u_{n}\right\}_{n \geq 0}$ and the initial condition $v_{0} \times \delta_{0}$ denotes the law of the pair $\left(X_{0}, Y_{0}\right)$. A p.o. control problem can be set once a cost functional is chosen. Then, we define a cost functional depending on the control $u$. and the initial conditions as

$$
J_{\text {p.o. }}\left(u_{.}, v_{0} \times \delta_{0}\right)=\mathrm{E}\left[\int_{0}^{T} c\left(t, X_{t}, u_{t}\right) \mathrm{d} t+g\left(X_{T}\right)\right] .
$$

The running cost $c(t, x, u)$ and the terminal cost $g(x)$ are nonnegative and bounded measurable functions and $c(t, x, u)=0$ if $|x|=0$. Let us observe that in (3.2) the integral can be computed either on $[0, T]$ or $\left[0, T \wedge \tau_{N}\right]$ because the state $x$, such that $|x|=0$, naturally, is an absorbing observable state for the process. A partially observable control problem reduces to minimizing a cost functional of the form (3.2) over $\mathcal{U}$, the set of all admissible controls.

We now introduce the separated control problem according to a standard procedure in the partially observed setup. Let us denote by $\pi_{t}$ the càdlàg version (that which is continuous from the right with left limits) of the conditional distribution of $X_{t}$ given $\mathcal{F}_{t}^{Y}$, defined, for any realvalued function $f$ on $\mathcal{X}$, as $\pi_{t}(f)=\mathrm{E}\left[f\left(X_{t}\right) \mid \mathcal{F}_{t}^{Y}\right]$. Since the $\left(\mathrm{P}, \mathcal{F}_{t}^{Y}\right)$-intensity (minimal 
intensity) of $Y_{t}$ is given by $\pi_{t}\left(\lambda_{1}^{u_{t}}\right), \pi_{t}$ is a solution of the controlled Kushner-Stratonovich equation (KS equation) [1, p. 83], i.e. for any real-valued function $f$ on $\mathcal{X}$,

$$
\begin{aligned}
\pi_{t}(f)= & v_{0}(f)+\int_{0}^{t} \pi_{s}\left(L^{u_{s}} f\right) \mathrm{d} s \\
& +\int_{0}^{t}\left(\pi_{s-}\left(L_{1}^{u_{s}} f\right)-\pi_{s-}(f) \pi_{s-}\left(\lambda_{1}^{u_{s}}\right)+\pi_{s-}\left(\lambda_{1}^{u_{s}} f\right)\right) \\
& \times\left(\pi_{s-}\left(\lambda_{1}^{u_{s}}\right)\right)^{+}\left[\mathrm{d} Y_{s}-\pi_{s-}\left(\lambda_{1}^{u_{s}}\right) \mathrm{d} s\right],
\end{aligned}
$$

where $a^{+}=\mathbf{1}_{(0,+\infty)}(a) / a$.

Moreover, the cost functional, (3.2), can be also written as

$$
\begin{aligned}
J_{\text {p.o. }}\left(u_{.}, v_{0} \times \delta_{0}\right) & =\mathrm{E}\left[\int_{0}^{T} \mathrm{E}\left[c\left(t, X_{t}, u_{t}\right) \mid \mathcal{F}_{t}^{Y}\right] \mathrm{d} t+\mathrm{E}\left[g\left(X_{T}\right) \mid \mathcal{F}_{T}^{Y}\right]\right] \\
& =\mathrm{E}\left[\int_{0}^{T} \pi_{t}\left(c\left(t, \cdot, u_{t}\right)\right) \mathrm{d} t+\pi_{T}(g(\cdot))\right] .
\end{aligned}
$$

Recall that a separated control problem is a sequence $\left(\Omega, \mathcal{F}_{t}, \mathrm{P}, \pi_{t}, Y_{t}, u_{t}, v_{0}, 0\right)$, where $\left(\Omega, \mathcal{F}_{t}, \mathrm{P}\right)$ is a filtered probability space, $u_{t}$ is an admissible control defined on it, and $(\pi, Y)$ is a weak solution of (3.3), i.e. $\left(\pi_{t}, Y_{t}\right)$ solves (3.3) and $\pi_{t}\left(\lambda_{1}^{u_{t}}\right)$ is the intensity of $Y_{t}, \pi_{0}=v_{0}$, and $Y_{0}=0$ P-a.s. The aim of the separated control problem is to minimize a cost functional of the form

$$
J_{S}\left(u_{.}, v_{0} \times \delta_{0}\right)=\mathrm{E}\left[\int_{0}^{T} \pi_{t}\left(c\left(t, ., u_{t}\right)\right) \mathrm{d} t+\pi_{T}(g(\cdot))\right] .
$$

Let us observe that in (3.5) $\pi$ is any solution of the KS equation, while in (3.4) $\pi$ is the conditional distribution of $X_{t}$ given $\mathcal{F}_{t}^{Y}$. Thus, it is quite intuitive that equivalence between the two problems is based on a result of uniqueness for the KS equation.

Uniqueness for the KS equation could be obtained by the filtering martingale problem approach presented in [20] and [21], as we did in [7]. But, the particular structure of the model presented in this paper allows us to use a more direct and easier method, which we discuss in the next section.

\section{The separated problem}

The main results of this section are the proofs of the equivalence between the partially observed control problem and the separated control problem.

Theorem 4.1. Under our setting, the equivalence between the partially observable control problem and the separated control problem holds in the sense that

$$
\inf J_{s}\left(u_{.}, v_{0}, 0\right)=\inf J_{\text {p.o. }}\left(u_{.}, v_{0}, 0\right),
$$

where the infimum is computed over all the admissible controls or, by (3.1), over all the families $\left\{u_{n}\right\}_{n \geq 0}$.

In order to prove this theorem, we note that, in general,

$$
\inf J_{s}\left(u, v_{0}, 0\right) \leq \inf J_{\text {p.o. }}\left(u_{.}, v_{0}, 0\right) .
$$

The converse inequality is a consequence of Theorem 4.2, Proposition 4.1, and Proposition 4.2, below. 
Theorem 4.2. The controlled KS equation, (3.3), has a unique pathwise solution. Moreover, such a solution is, necessarily, $\mathcal{F}_{t}^{Y}$-adapted for each admissible control.

Proof. At any jump time $\tau_{n}<\tau_{N}$, the filter is uniquely determined by the knowledge of $\pi_{\tau_{n}-} ;$ in fact,

$$
\pi_{\tau_{n}}(f)=\pi_{\tau_{n}-}\left(L_{1}^{u_{\tau_{n}}} f+\lambda_{1}^{u_{\tau_{n}}} f\right)\left[\pi_{\tau_{n}-}\left(\lambda_{1}^{u_{\tau_{n}}}\right)\right]^{+}
$$

and the thesis is trivial. For $t \in\left[\tau_{n}, \tau_{n+1}\right)$, with $\tau_{n+1} \leq \tau_{N}$,

$$
\pi_{t}(f)=\pi_{\tau_{n}}(f)+\int_{\tau_{n}}^{t}\left(\pi_{s}\left(L_{0}^{u_{s}} f\right)+\pi_{s}(f) \pi_{s}\left(\lambda_{1}^{u_{s}}\right)-\pi_{s}\left(\lambda_{1}^{u_{s}} f\right)\right) \mathrm{d} s,
$$

which is Lipschitz with respect to the bounded variation norm. Then, the solution is unique and necessarily $\mathcal{F}_{t}^{Y}$-adapted.

Proposition 4.1. Under our setting, weak uniqueness for the controlled KS equation, (3.3), holds.

Proof. The proof is a generalization of the proof of Theorem 4.5 of [5].

Given $\left\{u_{n}\right\}_{n \geq 0}$ and $\nu_{0}$, let the probability space $\left(\Omega,\left\{\mathcal{F}_{t}\right\}, \mathrm{P}\right)$ and the process $X_{t}$ be given as in the proof of Proposition 3.1, let $Y_{t}=N-\left|X_{t}\right|$, and let $\pi_{t}$ be the filter. Since strong uniqueness for (3.3) holds, then $\pi_{t}=\Psi\left(Y_{s}, s \leq t\right)$ P-a.s., where $\Psi: D_{\mathbb{N}}[0, T] \rightarrow \Pi(\mathcal{X})$ is a measurable function and $D_{\mathbb{N}}[0, T]$ is the space of right-continuous $\mathbb{N}$-valued functions on $[0, T]$ having left limits.

In the same way, let $(\tilde{\pi}, \widetilde{Y})$ be a solution of $(3.3)$, on the space $\left(\widetilde{\Omega},\left\{\widetilde{\mathcal{F}}_{t}\right\}, \widetilde{\mathrm{P}}\right)$, such that $\tilde{\pi}_{0}=v_{0}$

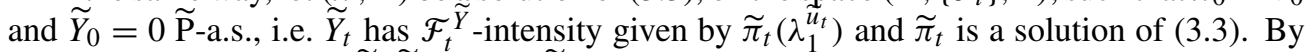
strong uniqueness, $\widetilde{\pi}_{t}=\widetilde{\Psi}\left(\widetilde{Y}_{s}, s \leq t\right) \widetilde{\mathrm{P}}$-a.s.

Since $Y_{t}$ has P-minimal intensity given by $\pi_{t}\left(\lambda_{1}^{u_{t}}\right)$ and $\widetilde{Y}_{t}$ has $\widetilde{\mathrm{P}}$-minimal intensity given by $\widetilde{\pi}_{t}\left(\lambda_{1}^{\widetilde{u}_{t}}\right)$, then $Y$, under $\mathrm{P}$, and $\widetilde{Y}$, under $\widetilde{\mathrm{P}}$, have the same law $[1$, p. 63], i.e. $(\pi, u, Y)$ and $(\tilde{\pi}, \tilde{u}, \widetilde{Y})$ have the same law.

Proposition 4.2. Under our setting and assuming weak uniqueness for the controlled KS equation, (3.3), given a separated control, there exists a partially observable control with the same cost.

Proof. The proof is again a generalization of the proof presented in Proposition 4.2 of [5]. Given a separated control $\left(\Omega, \mathcal{F}, \mathrm{P}, \pi_{t}, Y_{t}, u_{t}, v_{0}, 0\right)$ on the canonical space of the marked point processes for the same family $\left\{u_{n}\right\}_{n \geq 0}$ defining $u_{t}$, we construct a control $\widehat{u}_{t}$ defined by (3.1), a probability measure $\widehat{\mathrm{P}}$, and a process $\widehat{X}_{t}$ such that, under $\widehat{\mathrm{P}}, \widehat{X}_{t}$ is a solution of the controlled $\operatorname{MgP}\left(L^{\widehat{u}_{t}}, v_{0}\right)$, as in Proposition 3.1, and we set $\widehat{Y}_{t}=N-\left|\widehat{X}_{t}\right|$.

Let $\widehat{\pi}_{t}(f)=\mathrm{E}\left[f\left(\widehat{X}_{t}\right) \mid \widetilde{\mathcal{F}}_{t} \widehat{Y}\right]$. Then, $\widehat{\pi}_{t}$ solves the KS equation, (3.3), driven by $\widehat{Y}_{t}$. By uniqueness for this equation, the processes $\left(\pi_{t}, u_{t}, Y_{t}\right)$ and $\left(\widehat{\pi}_{t}, \widehat{u}_{t}, \widehat{Y}_{t}\right)$ have the same law under $\mathrm{P}$ and $\widehat{\mathrm{P}}$, respectively.

\section{Existence of an optimal control}

The separated control problem, whose state variable is the pair $\left(\pi_{t}, Y_{t}\right)$, is not yet a totally observed control problem. Actually, $Y$ and $\pi$ are not symmetric because $\pi_{t}$ is $\mathcal{F}_{t}^{Y}$-adapted and $u_{t}$ is $\mathcal{F}_{t}^{Y}$-predictable. In the control literature this is the reason why, by widening the class of controls, that is, by considering controls which are $\mathcal{F}_{t}^{\pi, Y}$-measurable, the separated generalized control problem is introduced. 
Remark 5.1. As a consequence of Theorem 4.2, we find that any solution of the filtering equation, (3.3), is necessarily $\mathcal{F}_{t}^{Y}$-adapted. Then, $\mathcal{F}_{t}^{\pi, Y}=\mathcal{F}_{t}^{Y}$ and the equivalence between the separated generalized problem and the separated problem obviously follows.

Thus, for our model, the introduction of generalized controls is not necessary. Furthermore, recalling that any measure $v$ on $\mathcal{X}$ is completely determined by the family of its atoms and that $\mathcal{X}$ is a finite set, the separated problem we are dealing with turns out to be a totally observed, finite-dimensional problem, which we will discuss later on.

In order to obtain the existence of an optimal control, we follow a classical procedure. Firstly, the set of admissible controls has to be widened to consider relaxed controls. More precisely, let $V$ denote the set of Radon measures on $[0, T] \times U$, whose projection on $[0, T]$ is the Lebesgue measure. Any element $r \in V$ can be split as $r(\mathrm{~d} s, \mathrm{~d} u)=r_{s}(\mathrm{~d} u) \mathrm{d} s$, where $r_{s}(\mathrm{~d} u)$ is a measurable kernel with mass equal to 1 . Endowing $V$ with the weak topology, since $U$ is compact, $V$ is a compact space. A relaxed control is an $\mathcal{F}_{t}^{Y}$-predictable random variable with values in $V$. A strictly admissible control process can be identified as a random variable with values in $V_{0}=\left\{r \in V: r(\mathrm{~d} s, \mathrm{~d} u)=\delta_{u_{s}}(\mathrm{~d} u) \mathrm{d} s\right\}$, the subset of atomic measures of $V$. A relaxed control $r$ is $\mathcal{F}_{t}^{Y}$-adapted or $\mathcal{F}_{t}^{Y}$-predictable if, for any bounded measurable function $h$ on $[0, T] \times U$, the process $\int_{0}^{t} \int_{U} h(s, u) r_{s}(\mathrm{~d} u) \mathrm{d} s$ is $\widetilde{\mathcal{F}}_{t}^{Y}$-adapted or, respectively, $\mathcal{F}_{t}^{Y}$-predictable. The relaxed separated control problem consists of minimizing the cost functional, (3.5), when the dynamics of $\pi_{t}$ are defined by the KS equation, (3.3).

Secondly, we observe that both (3.3) and (3.5) have to be modified according to the introduction of relaxed controls. In fact, the $\left(\mathrm{P}, \mathcal{F}_{t}\right)$-intensity of $Y_{t}$ is given by

$$
\lambda_{1}^{r_{s}}\left(X_{S}\right)=\int_{U} \lambda_{1}^{u}\left(X_{S}\right) r_{S}(\mathrm{~d} u)
$$

and the minimal intensity of $Y_{t}$, the $\left(\mathrm{P}, \mathcal{F}_{t}^{Y}\right)$-intensity, is given by $\pi_{t}\left(\lambda_{1}^{r_{t}}\right)$. Setting $L^{r_{s}} f=$ $L_{0}^{r_{s}} f+L_{1}^{r_{s}} f$ and $L_{i}^{r_{s}}=\int_{U} L_{i}^{u} f r_{s}(\mathrm{~d} u)$ for $i=0,1$, (3.3) and (3.5) become

$$
\begin{aligned}
\pi_{t}(f)= & v_{0}(f)+\int_{0}^{t} \pi_{s}\left(L^{r_{s}} f\right) \mathrm{d} s \\
& +\int_{0}^{t}\left(\pi_{s-}\left(L_{1}^{r_{s}} f\right)-\pi_{s-}(f) \pi_{s-}\left(\lambda_{1}^{r_{s}}\right)\right. \\
& \left.\quad+\pi_{s-}\left(\lambda_{1}^{r_{s}} f\right)\right)\left(\pi_{s-}\left(\lambda_{1}^{r_{s}}\right)\right)^{+}\left[\mathrm{d} Y_{s}-\pi_{s-}\left(\lambda_{1}^{r_{s}}\right) \mathrm{d} s\right]
\end{aligned}
$$

and

$$
J_{s}\left(r_{.}, \nu_{0} \times \delta_{0}\right)=\mathrm{E}\left[\int_{0}^{T} \int_{U} \pi_{t}(c(t, \cdot, u)) r_{t}(\mathrm{~d} u) \mathrm{d} t+\pi_{T}(g(\cdot))\right] .
$$

Finally, the desired result is a consequence of Proposition 5.1 and Theorem 5.1, below.

Proposition 5.1. Under our setting, the relaxed separated problem is equivalent to the original problem.

Proof. The thesis can be reached in three classical steps. First of all, the continuity of the criterion can be obtained with a procedure similar to the proof given in Proposition 2.11 of [25]. Then, the Chattering lemma [8] allows us the approximation of any relaxed control with a sequence of strictly admissible controls. Finally, for any relaxed control, the KS equation has a unique pathwise solution which is necessarily $\mathcal{F}_{t}{ }^{Y}$-adapted. This can be achieved by generalizing the proof of Theorem 4.2 to the case of relaxed controls 
Theorem 5.1. Under our setting, there exists an optimal relaxed separated control.

Proof. Since the continuity of criterion (3.5) holds, the thesis is a consequence of the compactness for $\left(\pi_{t}, Y_{t}, r_{t}\right)$ over its canonical space $D_{\Pi(X)}[0, T] \times D_{\mathbb{N}}[0, T] \times D_{V}[0, T]$, and, since $V$ is compact, only the compactness of the family $\left(\pi_{t}, Y_{t}\right)$ must be proven.

Step 1: Tightness. For $\pi_{t}$, only the tightness of $\pi_{t}(f)$ is necessary for all $f$ bounded [26]. Then, the tightness of $\left(\pi_{t}(f), Y_{t}\right)$ can be deduced by verifying the following two conditions (see Theorem 7.2 and Theorem 8.6 of [10]):

1. for all $\eta>0$ and $t>0$, there exists $M>0$ such that $\mathrm{P}\left(\left|\pi_{t}(f)\right|+Y_{t}>M\right)<\eta$;

2. for all $T>0$ and $0<\delta<1$, there exists nonnegative random variables $\zeta(\delta)$ such that $\lim _{\delta \rightarrow 0} \sup _{r_{s}} \mathrm{E}[\zeta(\delta)]=0$ and

$$
\mathrm{E}\left[\left|\pi_{t+v}(f)-\pi_{t}(f)\right|+Y_{t+v}-Y_{t} \mid \mathcal{F}_{t}^{Y}\right] \leq \mathrm{E}\left[\zeta(\delta) \mid \mathcal{F}_{t}^{Y}\right] \quad \text { for all } v \leq \delta .
$$

The first condition is easily verified for big enough $M$, since $\mathrm{E}\left[\left|\pi_{t}(f)\right|+Y_{t} \mid \mathcal{F}_{t}^{Y}\right] \leq\|f\|+$ $T \Lambda(4\|f\|+1)$, where we define $\Lambda$ such that

$$
\sup _{u, x} \lambda_{0}^{u}(x)+\sup _{u, x} \lambda_{1}^{u}(x) \leq \Lambda \quad \text { for all } u \in U \text { and } x \in \mathcal{X} .
$$

Moreover, as a consequence of (5.1),

$$
\begin{aligned}
& \mathrm{E}\left[\left|\pi_{t+u}(f)-\pi_{t}(f)\right|+Y_{t+u}-Y_{t} \mid \mathcal{F}_{t}^{Y}\right] \\
& \quad \leq \mathrm{E}\left[\int_{t}^{t+v}\left(\pi_{s}\left(\left|L_{0}^{r_{s}} f\right|+\left|L_{1}^{r_{s}} f\right|\right)+\left(2 \pi_{s}(|f|)+1\right) \pi_{s}\left(\lambda_{1}^{r_{s}}\right)+2 \pi_{s}\left(\lambda_{1}^{r_{s}}|f|\right)\right) \mathrm{d} s \mid \mathcal{F}_{t}^{Y}\right],
\end{aligned}
$$

and the choice $\zeta(\delta)=\delta \Lambda(4\|f\|+1)$ satisfies the second condition.

Step 2: Closure. On the canonical space $D_{\Pi(\mathcal{X})}[0, T] \times D_{\mathbb{N}}[0, T] \times D_{V}[0, T]$ endowed with its canonical filtration $\varepsilon_{t}$, let $\mathbb{P}^{k} \Rightarrow \mathbb{P}^{\infty}$ be a convergent subsequence of the tight family $\{\mathbb{P}\}$. To identify $\mathbb{P}^{\infty}$, we have to prove that it provides a solution to the controlled martingale problem for the operator

$$
\begin{aligned}
& \mathcal{B}^{r_{s}} F(\pi(f), y) \\
& =F_{p}^{\prime}(\pi(f), y)\left(\pi\left(L_{0}^{r_{s}} f\right)+\pi(f) \pi\left(\lambda_{1}^{r_{s}}\right)-\pi\left(\lambda_{1}^{r_{s}} f\right)\right) \\
& \quad+\pi\left(\lambda_{1}^{r_{s}}\right)\left(F\left(\pi(f)+\pi\left(\lambda_{1}^{r_{s}}\right)^{+}\left\{\pi_{s}\left(L_{1}^{r_{s}} f\right)-\pi_{s}(f) \pi_{s}\left(\lambda_{1}^{r_{s}}\right)+\pi_{s}\left(\lambda_{1}^{r_{s}} f\right)\right\}, y+1\right)\right. \\
& \quad-F(\pi(f), y)) .
\end{aligned}
$$

We know that, for all $F(p, d)$ such that $F$ itself and its first derivative $F_{p}^{\prime}$ are bounded and continuous functions,

$$
\mathbb{M}_{t}^{r_{t}}(\pi(\cdot), Y)=F\left(\pi_{t}(f), Y_{t}\right)-F\left(\nu_{0}(f), 0\right)-\int_{0}^{t} \mathcal{B}^{r_{s}} F\left(\pi_{s}(f), D_{s}\right) \mathrm{d} s
$$

is an $\left(\varepsilon_{t}, \mathbb{P}^{k}\right)$-martingale. Thus, for all $t_{0}<t$ and all $g_{t_{0}}$ continuous and bounded on $D_{\Pi(\mathcal{X})}[0, T] \times D_{\mathbb{N}}[0, T], \mathcal{E}_{t_{0}}$-measurable, $\mathbb{E}^{\mathbb{P}^{k}}\left[\left(\mathbb{M}_{t}^{r .}-\mathbb{M}_{t_{0}}^{r_{0}}\right) g_{t_{0}}\right]=0$. Since $\left(\mathbb{M}_{t}^{r .}-\mathbb{M}_{t_{0}}^{r_{0}}\right) g_{t_{0}}$ is a bounded continuous functional of its arguments, $\mathrm{E}^{\mathbb{P} \infty}\left[\left(\mathbb{M}_{t}^{r \cdot}-\mathbb{M}_{t_{0}}^{r}\right) g_{t_{0}}\right]=0$, which means that $\mathbb{M}_{t}^{r}$ is an $\left(\varepsilon_{t}, \mathbb{P}^{\infty}\right)$-martingale. This implies that $\pi_{t}(f)-\pi_{0}(f)-\int_{0}^{t} \pi_{s}\left(L^{r_{s}} f\right) \mathrm{d} s$ is an $\left(\varepsilon_{t}, \mathbb{P}^{\infty}\right)$-martingale with bounded variation and bounded jumps, and the same holds for $Y_{t}-\int_{0}^{t} \pi_{s}\left(\lambda_{1}^{r_{s}}\right) \mathrm{d} s$. Finally, by Proposition 14.65 of [18], $\left(\pi_{t}, Y_{t}\right)$ is a solution of the KS equation for the relaxed controls. Then, the thesis is a consequence of the pathwise uniqueness for (5.1). 


\section{Finite-dimensional representation and discrete-time approximation}

In this section we study the separated problem. The discussions in the previous sections allow us to claim that this is a controlled, totally observed problem. The state variables are $Y_{t}$ and $\pi_{t}$, and their dynamics, under the action of a constant control $u$, for any bounded measurable function $F$ which is continuous with its first derivative with respect to its first variable, is described by the operator

$$
\begin{gathered}
\mathscr{B}^{u} F(\pi(f), y)=F_{p}^{\prime}(\pi(f), y)\left(\pi\left(L_{0}^{u} f\right)+\pi(f) \pi\left(\lambda_{1}^{u}\right)-\pi\left(\lambda_{1}^{u} f\right)\right) \\
+\pi\left(\lambda_{1}^{u}\right)\left(F\left(\pi(f)+\pi\left(\lambda_{1}^{u}\right)^{+}\left\{\pi_{s}\left(L_{1}^{u} f\right)-\pi_{s}(f) \pi_{s}\left(\lambda_{1}^{u}\right)+\pi_{s}\left(\lambda_{1}^{u} f\right)\right\}, y+1\right)\right. \\
-F(\pi(f), y)) .
\end{gathered}
$$

The first problem we are faced with is that, in general, $\pi$ is not finite dimensional. In our setting, this is a false problem, since $\pi$ is purely atomic with a finite number of atoms and it is completely described by the value on its atoms.

Therefore, our problem turns out to be a finite-dimensional, totally observed control problem. The state variables are $Y_{t}$ and the vector $\Pi_{t}=\left\{\Pi_{t}(x), x \in \mathcal{X}\right\}=\left\{\pi_{t}\left(\mathbf{1}_{\{=x\}}\right), x \in \mathcal{X}\right\}$, which takes values in the set

$$
s:=\left\{\{\Pi(x)\}_{x \in \mathcal{X}}, \Pi(x) \geq 0, \sum_{x \in \mathcal{X}} \Pi(x)=1\right\} \subset[0,1]^{x} .
$$

Defining the vector $\Lambda_{1}^{u_{t}}:=\left\{\lambda_{1}^{u_{t}}(x), x \in \mathcal{X}\right\}$, the minimal intensity of $Y$ can be written as

$$
\pi_{t}\left(\lambda_{1}^{u_{t}}(\cdot)\right):=\left(\Lambda_{1}^{u_{t}}, \Pi_{t}\right)=\sum_{x \in \mathcal{X}} \lambda_{1}^{u_{t}}(x) \Pi_{t}(x)
$$

Moreover, the KS equation becomes

$$
\Pi_{t}=\Pi_{0}+\int_{0}^{t} b\left(\Pi_{s}, u_{s}\right) \mathrm{d} s+\int_{0}^{t} a\left(\Pi_{s-}, u_{s}\right) \mathrm{d} Y_{s},
$$

where the vectors $\Pi_{0}, b$, and $a$ are given by

$$
\begin{aligned}
\Pi_{0} & =\left\{\Pi_{0}(x), x \in \mathcal{X}\right\}=\left\{v_{0}\left(\mathbf{1}_{\{=x\}}\right), x \in \mathcal{X}\right\}, \\
b\left(\Pi_{s}, u_{s}\right)(x) & =\sum_{x^{\prime} \in \mathcal{X}} \lambda_{0}^{u_{s}}\left(x^{\prime}\right) \mu_{0}^{u_{s}}\left(x^{\prime}, x\right) \Pi_{s}\left(x^{\prime}\right)+\left(\lambda_{1}^{u_{s}}, \Pi_{s}\right) \Pi_{s}(x)-\left(\lambda_{0}^{u_{s}}(x)+\lambda_{1}^{u_{s}}(x)\right) \Pi_{s}(x), \\
a\left(\Pi_{s}, u_{s}\right)(x) & =\left(\sum_{x^{\prime} \in \mathcal{X}} \lambda_{1}^{u_{s}}\left(x^{\prime}\right) \mu_{1}^{u_{s}}\left(x^{\prime}, x\right) \Pi_{s}\left(x^{\prime}\right)-\left(\Lambda_{1}^{u_{s}}, \Pi_{s}\right) \Pi_{s}(x)\right)\left(\Lambda_{1}^{u_{s}}, \Pi_{s}\right)^{+} .
\end{aligned}
$$

Furthermore, introducing the vectors

$$
c\left(t, u_{t}\right):=\left\{c\left(t, x, u_{t}\right), x \in \mathcal{X}\right\} \quad \text { and } \quad g:=\{g(x), x \in \mathcal{X}\},
$$

and setting

$$
\left(c\left(t, u_{t}\right), \Pi_{t}\right)=\sum_{x \in \mathcal{X}} c\left(t, x, u_{t}\right) \Pi_{t}(x) \quad \text { and } \quad\left(g, \Pi_{T}\right)=\sum_{x \in \mathcal{X}} g(x) \Pi_{T}(x),
$$


the cost functional, (3.5), can be written as

$$
J_{s}\left(u_{.}, v_{0} \times \delta_{0}\right)=\mathrm{E}\left[\int_{0}^{T}\left(c\left(t, u_{t}\right), \Pi_{t}\right) \mathrm{d} t+\left(g, \Pi_{T}\right)\right] .
$$

At this point, we construct a suitable discrete-time approximation. This can be done in several ways. For instance, a discrete-time system converging to the original one was constructed in [6] and [12], generalizing some of the results presented in [2] and [3]. But, an essential tool in the procedure used there was the homogeneity with respect to time of the processes involved. Thus, the same procedure cannot be adapted to our system, where the dynamics are controlled and, in general, are nonhomogeneous with respect to time.

Then, we will use a procedure related to the well-known classical methods pioneered in [23] and [24, Chapter 10] (see also the references therein). For the sake of self-consistency, we provide a sketch of the procedure, but we refer the reader to the abovementioned papers for any further detail.

Taking into account (6.1), for a constant control $u \in U$, we find that the dynamics of the process $\left(\Pi_{t}, Y_{t}\right)$ can be described by the operator

$$
\begin{aligned}
\mathcal{L}^{u} f(\Pi, y) & =\left(\nabla_{\Pi} f, b(\Pi, u)\right)+\left(\lambda_{1}^{u}, \Pi\right)(f(\Pi+a(\Pi, u), y+1)-f(\Pi, y)) \\
& =\sum_{x \in \mathcal{X}} \frac{\partial f}{\partial \Pi(x)} b(\Pi, u)(x)+\left(\lambda_{1}^{u}, \Pi\right)(f(\Pi+a(\Pi, u), y+1)-f(\Pi, y)) .
\end{aligned}
$$

Remark 6.1. Let us note that, since $\sum_{x \in \mathcal{X}} b\left(\Pi_{s}, u_{s}\right)(x)=0$ and $\sum_{x \in \mathcal{X}} a\left(\Pi_{s}, u_{s}\right)(x)=0$, $\Pi+h b(\Pi, u)$ and $\Pi+a(\Pi, u)$ still belong to $\&$ if $\Pi$ does.

For $h>0$, we consider the finite-difference operator

$\mathcal{L}_{h}^{u} f(\Pi, y)=\frac{f(\Pi+h b(\Pi, u), y)-f(\Pi, y)}{h}+\left(\lambda_{1}^{u}, \Pi\right)(f(\Pi+a(\Pi, u), y+1)-f(\Pi, y))$

and the controlled Markov chain $\left(\Pi_{n}^{h}, Y_{n}^{h}\right)$ with transition probabilities given, for constant controls, by

$$
\begin{gathered}
p^{h}((\Pi, y),(\Pi+h b(\Pi, u), y) \mid u)=\frac{1 / h}{1 / h+\left(\lambda_{1}^{u}, \Pi\right)}=\frac{1}{1+\left(\lambda_{1}^{u}, \Pi\right) h}, \\
p^{h}((\Pi, y),(\Pi+a(\Pi, u), y+1) \mid u)=\frac{\left(\lambda_{1}^{u}, \Pi\right)}{1 / h+\left(\lambda_{1}^{u}, \Pi\right)}=\frac{\left(\lambda_{1}^{u}, \Pi\right) h}{1+\left(\lambda_{1}^{u}, \Pi\right) h},
\end{gathered}
$$

where the $p^{h}((\Pi, y),(\widetilde{\Pi}, \tilde{y}) \mid u)$ are 0 for all nonlisted values of $\widetilde{\Pi}$ and $\tilde{y}$.

The class of admissible controls for $\left(\Pi_{n}^{h}, Y_{n}^{h}\right)$ is given by the families $\left\{u_{n}^{h}\right\}_{n \geq 0}$ of $U$-valued random variables preserving the Markov property.

Then, we define the time interpolation interval

$$
\Delta t^{h}(\Pi, u):=\frac{h}{1+\left(\lambda_{1}^{u}, \Pi\right) h} .
$$

Definition 6.1. Let the interpolated continuous-time process $\left(\Pi^{h}(\cdot), Y^{h}(\cdot)\right)$ and control $u^{h}(\cdot)$ be defined by

$$
Y^{h}(t):=Y_{n}^{h}, \quad \Pi^{h}(t):=\Pi_{n}^{h}, \quad \text { and } \quad u^{h}(t):=u_{n}^{h}
$$

on $t \in\left[t_{n}^{h}, t_{n+1}^{h}\right)$, where $t_{n}^{h}:=\sum_{i=0}^{n-1} \Delta t^{h}\left(\Pi_{i}^{h}, u_{i}^{h}\right)$. 
Recalling (6.2), let us define the cost for the original problem, when the initial condition is $(\Pi, t)$ for $t \geq 0$ and $\Pi \in \delta$, as

$$
J(r, \Pi, t)=\mathrm{E}\left[\int_{t}^{T} \int_{U}\left(c(s, \alpha), \Pi_{s}\right) r_{s}(\mathrm{~d} \alpha) \mathrm{d} s+\left(g, \Pi_{T}\right)\right],
$$

where $r(\cdot)$ is an admissible relaxed control for the original process and, analogously, let the cost for the approximated problem be defined as

$$
J^{h}\left(r^{h}, \Pi, t\right)=\mathrm{E}\left[\int_{t}^{T} \int_{U}\left(c(s, \alpha), \Pi^{h}(s)\right) r_{s}^{h}(\mathrm{~d} \alpha) \mathrm{d} s+\left(g, \Pi^{h}(T)\right)\right],
$$

where $r_{t}^{h}(\mathrm{~d} \alpha):=\delta_{u_{t}^{h}}(\mathrm{~d} \alpha)$ is the relaxed control for the approximated system.

Finally, if we define the optimal cost for the original and approximated problems as

$$
V(\Pi, t):=\inf _{\{r .\}} J(r ., \Pi, t) \text { and } V^{h}(\Pi, t):=\inf _{\left\{r_{.}^{h}\right\}} J^{h}\left(r^{h}, \Pi, t\right),
$$

respectively, we can claim, taking account of the results presented in [24, Chapter 10], that the approximating system $\left(\Pi^{h}(\cdot), Y^{h}(\cdot), r_{t}^{h}(\cdot)\right)$ converges weakly to the original system and, moreover, that

$$
V^{h}(\Pi, t) \longrightarrow V(\Pi, t) \quad \text { as } h \rightarrow 0 .
$$

\section{References}

[1] Brémaud, P. (1980). Point Processes and Queues. Springer, Berlin.

[2] Calzolari, A. AND NAPPo, G. (1996). A filtering problem with counting observations: approximations with error bounds. Stoch. Stoch. Reports 57, 71-87.

[3] Calzolari, A. and NapPo, G. (1997). A filtering problem with counting observations: error bounds due to the uncertainty on the infinitesimal parameters. Stoch. Stoch. Reports 61, 1-19.

[4] Ceci, C. And Gerardi, A. (1998). Partially observed control of a Markov jump process with counting observations: equivalence with the separated problem. Stoch. Process. Appl. 78, 245-260.

[5] Ceci, C., Gerardi, A. and Tardelli, P. (2001). An approximation method for partially observed controlled discrete jump Markov processes. IEEE Trans. Automatic Control 46, 1850-1861.

[6] Ceci, C., Gerardi, A. And Tardelli, P. (2001). An estimate of the approximation error in the filtering of a discrete jump process. Math. Models Meth. Appl. Sci. 2, 181-198.

[7] Ceci, C., Gerardi, A. and Tardelli, P. (2002). Existence of optimal control for partially observed jump processes. Acta Appl. Math. 74, 155-175.

[8] El Karoui, N., Huu Nguyen, D. and Jeanblanc-Picqué, M. (1988). Existence of an optimal Markovian filter for control under partial observations. SIAM J. Control Optimization 26, 1025-1061.

[9] Elliott, R. J., Aggoun, L. And Moore, J. B. (1995). Hidden Markov Models: Estimation and Control. Springer, New York.

[10] Ethier, S. N. And Kurtz, T. G. (1986). Markov Processes: Characterization and Convergence. John Wiley, New York.

[11] Fleming, W. (1980). Measure-valued processes in the control of partially-observable stochastic systems. Appl. Math. Optimization 6, 271-285.

[12] Gerardi, A. and Tardelli, P. (2001). Finite state and discrete time approximation for filters. Nonlinear Anal. Ser. A 47, 2485-2495.

[13] Gerardi, A. And Tardelli, P. (2005). Heterogeneous population dynamical model: a filtering problem. J. Appl. Prob. 42, 346-361.

[14] Gerardi, A., Spizzichino, F. And Torti, B. (2000). Exchangeable mixture models for lifetimes: the role of 'occupation number'. Statist. Prob. Lett. 49, 365-375.

[15] Gerardi, A., Spizzichino, F. and Torti, B. (2000). Filtering equations for the conditional law of residual lifetimes from a heterogeneous population. J. Appl. Prob. 37, 823-834.

[16] Hernandez-Hernandez, D., Marcus, S. I. And Fard, P. J. (1999). Analysis of a risk-sensitive control problem for hidden Markov chains. IEEE Trans. Automatic Control 44, 1093-1100. 
[17] JACOD, J. (1975). Multivariate point processes: predictable projection. Z. Wahrscheinlichkeitsth. 31, $235-253$.

[18] JACOD, J. (1979). Calcul Stochastique et Problèmes de Martingale. Springer, Berlin.

[19] James, M. R., BARA, J. S. AND Elliott, R. J. (1994). Risk-sensitive control and dynamic games for partially observed discrete-time nonlinear systems. IEEE Trans. Automatic Control 39, 780-791.

[20] Kurtz, T. G. (1998). Martingale problems for conditional distribution of Markov processes. Electron. J. Prob. 3, 1-29.

[21] Kurtz, T. G. AND Ocone, D. (1988). Unique characterization of conditional distributions in nonlinear filtering. Ann. Prob. 16, 80-107.

[22] Kurtz, T. G. and Stockbridge, R. H. (1998). Existence of Markov controls and characterization of optimal Markov controls. SIAM J. Control Optimization 36, 609-653.

[23] Kushner, H. J. (1990). Numerical methods for stochastic control problems in continuous time. SIAM J. Control Optimization 28, 999-1048.

[24] Kushner, H. J. And Dupuis, P. G. (1992). Numerical Methods for Stochastic Control Problems in Continuous Time. Springer, Berlin.

[25] MazliaK, L. (1992). Mixed control problem under partial observation. Appl. Math. Optimization 27, $57-84$.

[26] Roelly-Coppoletta, S. (1986). A criterion of convergence of measure valued processes: application to measure-branching processes. Stochastics 7, 44-66.

[27] TARdELli, P. (2006). Conditional law of lifetimes for a heterogeneous population of living particles via filtering techniques. Sci. Math. Japan 64, 915-932. 